\title{
Behavior shaping in studies of preweaning stress'
}

NORMAN D. HENDERSON

OBERLIN COLLEGE

To test whether early shock stress shapes locomotor behavior and thus confounds later "emotionality" measures based on exploration and escape learning, 64 Wistar rats in matched pairs were either restricted or allowed to move about freely during early shock. Age, sex, intensity and type of shock were cxamined. Results suggested that behavior shaping is not an important factor in early experience studies using open field, emergence, and escape-learning tests.

A common procedure in early stress studies with mice and rats is to administer electric shock prior to weaning and later test "emotionality" using open field, timidity, escape, and avoidance-learning measures. It has been suggested (Brookshire et al, 1961; Henderson, 1963, 1965) that one of the factors that influence the outcome of early experience studies is instrumental conditioning. Since many of the indices of emotional behavior involve ambulatory and running activity, the possibility should be examined that many altered behaviors found after early stress are the result of prior shaping of locomotor activity, rather than changes in "timidity" or "emotionality."

Method

Ss were 42 male and 42 female Wistar rats weaned at 24 days. The shock apparatus consisted of a box, divided into four equal 10-in. by 6 -in. by 7 -in. compartments with a clear plastic front and cover and a stainless steel grid. The grid in each compartment was wired independently to a 1150-VAC shock source in series with either a 1.0- or a 4.4-megohm resistor, providing shock levels of $1.00 \mathrm{ma}$ and $0.25 \mathrm{ma}$ respectively. Two sizes of restraining boxes were used to restrict Ss' gross activity during shock. The smaller box, used for 4- to 8-day Ss, was 1 in. by 2 in. by 1 in. high; while the box used for 19- to 23-day Ss was 2 in. by $3 \mathrm{in}$, by $2 \mathrm{in}$. high. Both were made of clear plastic and held down by weights.

Two identical open fields, 36 in. square, with 24-in. black walls were used. The floor of each field was divided into four equal 18-in. quadrants by two photoelectric beams intersecting at right angles. When $\mathrm{S}$ crossed either beam a count was registered. Suspended $18 \mathrm{in}$. above the center of the field was a red reflector floodlamp which provided a light of approximately 100

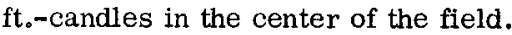

The emergence apparatus consisted of a 12-in. by 4-in. by 9-in. start box which opened by means of a guillotine door into a 30-in. runway of the same height and width. The start box was enclosed with solid floor, walls and cover. The runway was covered with wire mesh and opened into a brightly lighted 17-in.by 13-in. area. It was marked off in 5-in. units and $S$ could receive a distance-emerged score from 0 , if it did not leave the start box, to 7 if it traversed the complete runway.

The avoidance shuttlebox was 33 in. by $5-1 / 2$ in. by 16 in. high, divided into two equal compartments separated by a 1 -in. barrier. The grid floor was wired to a 240-VAC shock source which provided a .75-ma shock. The box was painted flat black except for the glass wall used for observation.

A matched pairs design was used so that for each "free" S, a like-sex littermate was given treatment simultaneously in the shock apparatus but was prevented with the restraining box from making any gross movements. The pair was weaned and caged together until testing, at which time they were tested simultaneously in the open fields; and one immediately followed the other in the emergence runway and the avoidance shuttlebox. To provide a variety of conditions under which shaping might occur, four between-pair factorseach at two levels-were studied. Intensity of shock (1.00 vs. $0.25 \mathrm{ma}$ ) and type of shock (continuous vs. intermittent) were the stress factors, and age at time of shock (4-8 days vs, 19-23 days) and sex were the two subject factors. Intermittent shock consisted of 1-sec. pulses every $30 \mathrm{sec}$. The shock treatments were repeated daily for five consecutive days, after which Ss were undisturbed until weaning and adult testing. Complete litters were arbitrarily chosen to receive shock from 4-8 days or from 19-23 days of age. Within each litter matched pairs were distributed among the four combinations of shock conditions and marked by toe clipping. In addition to the 64 shocked Ss, 20 nontreated Ss were reserved for later testing.

At days 35 and 36 Ss were tested in the open fields for $5 \mathrm{~min}$. in matched pairs. On Day $37 \mathrm{Ss}$ were placed in the start box of the emergence runway, and $\mathrm{E}$ recorded latency to enter the runway and the farthest line crossed in $3 \mathrm{~min}$. Following the emergence test, Ss were tested in the avoidance shuttlebox. E recorded the latency for $S$ to first cross the barrier between sections of the shuttlebox and the number of barrier crossings during a 2-min. period. The light was then turned on in the section opposite the one in which $\mathrm{S}$ was located, and $9.6 \mathrm{sec}$. later the grid on the dark side delivered a $.75 \mathrm{ma}$ shock to $\mathrm{S}$. This procedure was repeated for five trials, and the median escape latency was determined.

\section{Results and Discussion}

The number of fecal boluses on each day was transformed to the form $\sqrt{\mathrm{X}+.5}$, and the sum of the transformed scores for both days was the open field defecation score. Similarly, square roots of the number of 
Table 1. Summary of $F$ Ratios of Significant Restriction Effects

\begin{tabular}{lcccc} 
Source & Defecation & Ambulation & Emergence & Crosses \\
\hline Restriction & & $4.4^{*}$ & & \\
Res X Age 9.9 & 6.6 & 6.5 & \\
Res X Sex & & & 8.3 & 6.3 \\
\hline
\end{tabular}

$* p<.05 ;$ all others, $p<.02$

$d f=1 \cdot 2$ in each case

photocell counts made on each day were added to form the ambulation score.

Of major interest was the effect of restricting Ss during early shock. Table 1 presents the summary of significant restriction effects and interactions on the defecation, ambulation, distance-emerged, and barriercrossed measures. The three $\log$ latency measures showed no significant restriction effects. Most predominant was the significant interaction between age and restriction, which occurred in three of the dependent variables. Figure 1 shows the means of the free and restricted groups at the two age levels for the dependent variables summarized in Table 1 . The means for the
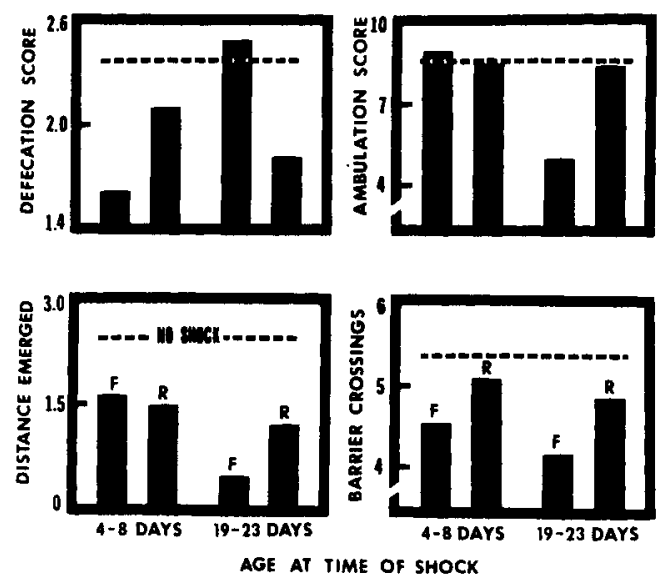

Fig. 1. Restriction by Age effects for defecation, ambulation, emergence, and barrier-cross measures.
20 undisturbed Ṡs are also shown. No significant differences occurred between free and restricted groups on any measure with young Ss. Older restricted Ss had significantly greater mean activity and distanceemerged scores, and significantly lower defecation scores than free Ss. The Restriction by Sex interaction on the emergence and barrier-cross measures was due to restricted females venturing further in the runway and crossing the barrier more frequently than free females, with no difference between males.

Compared to the 64 shocked Ss, the group of nonshocked controls emerged significantly further and had shorter starting latencies in the runway $(p<.02$ in both cases), made more barrier crosses $(p<.02)$, and had shorter latencies to cross the barrier initially $(p<.10)$.

There is no evidence in the present study that Ss free to move about during early shock subsequently show greater locomotor behavior than restrained Ss. Restricted Ss shocked from 19 to 23 days were more active in the open field and emerged further in the runway than free Ss. The significantly higher defecation score of the free Ss suggests, however, that the group was generally more fearful than the restrained Ss. Greater generalization of conditioned fear might be expected in the free group because of the greater similarity between the shock situation and the test situations. With Ss shocked from 4 to 8 days, none of the factors studied were significant.

\section{References}

Brookshire, K. H., Littman, R, A., \& Stewart, C, N. Residua of shock-trauma in the white rat: A three-factor theory, Psychol. Monogr., 1961, 75. No. 10 (Whole No. 514).

Henderson, N. D. Effect of prior runway experience on avoidance learning. Psychol. Rep., 1963, 13, 575-582.

Henderson, N. D. Acquisition and retention of conditioned fear during different stages in the development of mice. J. comp. physiol. Psychol., 1965, 59, 439-442.

\section{Note}

1. This study was supported by Grant NSF GB-1863 from the National Science Foundation. Mr. Michael Greene aided in the collection of these data. 